\section{SAT0050 STANDARDISATION OF SYNOVIAL BIOPSIES ANALYSIS: A EULAR SYNOVITIS STUDY GROUP INITIATIVE USING A DELPHI SURVEY.}

A. Najm ${ }^{1}$, B. Le Goff ${ }^{1}$, C. Orr ${ }^{2}$, J. Fonseca ${ }^{3}$, B. Lauwerys ${ }^{4}$, D. Veale ${ }^{2}$ on behalf of EULAR Synovitis Study Group. ${ }^{1}$ Rheumatology, Nantes University Hospital, NANTES, France, ${ }^{2}$ Centre for Arthritis and Rheumatic Disease, University College Dublin, Dublin Academic Medical Centre, St. Vincent's University Hospital., Dublin, Ireland, ${ }^{3}$ Hospital de Santa Maria \& Instituto de Medicina Molecular, Faculdade de Medicina da Universidade de Lisboa, Lisbon, Portugal, ${ }^{4}$ Université catholique de Louvain and Department of Rheumatology, Cliniques Universitaires Saint-Luc, Louvain, Belgium

Background: Synovial biopsies are increasingly performed in both clinical setting and translational research. ${ }^{1}$ An unmet need in the field is the standardisation of synovial biopsies handling and analysis procedures.

Objectives: The aim of this collaborative work was to create a consensual set of points to consider for handling and analysis of synovial biopsies in clinical practice and translational research through EULAR Synovitis Study Group (ESSG).

Methods: The items were identified and formulated based on a comprehensive literature review. ${ }^{1}$ A task force (TF) of EULAR Synovitis Study Group (ESSG) members were constituted and TF members were consulted through a 2-stage eDelphi process. the 2 sequential rounds occurred in 9 months. The first written round occurred in June 2016. Members were sent a written questionnaire containing items divided in 2 parts. The items were identified and formulated based on a comprehensive literature review. The first part of the questionnaire referred to clinical practice containing 5 subsections: biopsy sampling, biopsy handling, histological analysis, staining and immunohistochemistry $(\mathrm{IHC})$, biopsy analysis and pathologist's report. The second part referred to translational research and contained 6 subsections (same 5 plus RNA analysis). Every participant was asked to score each item with a 5 points Likert (0: strongly disagree, 5 : strongly agree), comments were allowed for each item. Items with a median score above 3.5 on 5 and a percentage of agreement above $70 \%$ were for the next round. Items with lower score were either suppressed of modified according to participants' comments. Anonymized detailed results were circulating through participants between each round. The face to face meeting occurred at ESSG meeting in June 2017. Results: 27 ESSG members from 19 centres were contacted by email. 20 participants from 17 centres answered (response rate of $74 \%$ ). Response rates for next rounds were $100 \%$. First questionnaire contained 44 items for Part 1 Clinical practice. $52.3 \%(23 i t e m s / 44)$ were selected for the second round based on their score and agreement percentage. $83 \%$ (19items/23) were selected for the third round. First questionnaire contained 43 items for the second part about translational research. 44\% (19 items/43) were selected for second round based on their score and agreement percentage. $95 \%$ (18 items/19) were selected for third round (figure 1). Third oral round allowed to obtain a final set of items unanimously (table 1).

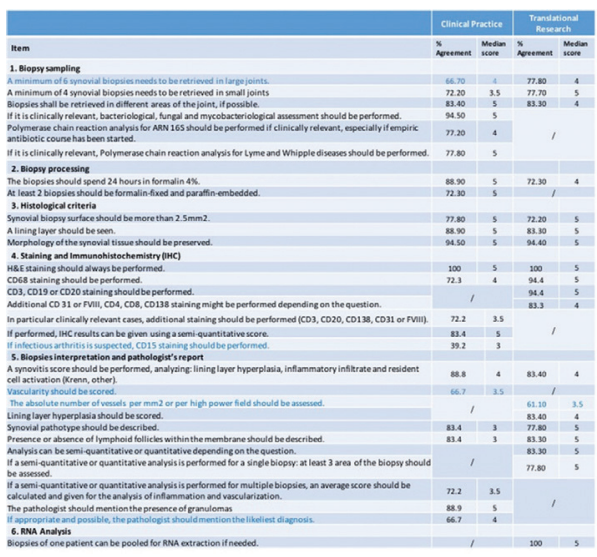

Conclusions: We hereby propose a set of consensual points to consider on analysis of synovial biopsies in clinical practice and translational research. This standardisation initiative was conducted through ESSG members using a validated consensus method.

\section{REFERENCE:}

[1] Orr C, Sousa E, Boyle DL, Buch MH, Buckley CD, Cañete JD, et al. Synovial tissue research: a state-of-the-art review. Nat Rev Rheumatol 2017;13 (8):463-75.

Disclosure of Interest: None declared DOI: 10.1136/annrheumdis-2018-eular.1624

\section{SAT0051 EXAMINING THE ROLE OF TRPC5 IN OSTEOARTHRITIS PAIN}

J. Sousa-Valente, F. Ayaz, X. Kodji, S.D. Brain. Vascular Biology and Inflammation Section, King's College London, London, UK

Background: Transient receptor potential canonical 5 (TRPC5) is a cation channel that putatively acts as a sensor of one or more chemical factors but the exact biology is not fully known. It was recently found to be expressed in fibroblast-like synoviocytes where TRPC5 activation by endogenous thioredoxin - which has been shown to be elevated in synovial fluid of patients with rheumatoid arthritis results in the suppression of matrix metalloproteinases secretion. ${ }^{1}$ Genetic deletion or pharmacological blockade of TRPC5 receptor in arthritic mice resulted in marked exacerbation of hyperalgesia and critically increased localised inflammation in the synovium, characterised by increased cellular infiltration, secretion of early response cytokines and enhanced synovial vascularisation. ${ }^{2}$ Interestingly, in this study, mRNA expression of TRPC5 was reduced in inflamed human arthritis samples particularly in the synovium from patients with osteoarthritis (OA), highlighting a potential role for TRPC5 in OA.

Objectives: With the aim to investigate putative mechanisms mediated by TRPC5 signalling in OA pain, we have examined the development of monoiodoacetate (MIA)-referred mechanical hypersensitivity and joint pathology in wild type and mice carrying a TRPC5 deletion.

Methods: Wild type and TRPC5 knock-out (TRPC5) mice were intra-articularly injected with $0.75 \mathrm{mg}$ of MIA, and hind-paw mechanical thresholds and weight bearing changes were assessed at regular intervals up to 28 days. Knee joint histo chemical analysis of cartilage degradation using toluidine blue staining and synovial inflammation using haematoxylin and eosin staining was also performed. Results: Both TRPC5 KO and WT mice developed referred mechanical hypersensitivity following MIA injection, however the onset was faster (day 3 vs day 10) in TRPC5 KO mice. By the end of the study, at 28 days after MIA injection, mechanical hypersensitivity had reached similar levels in TRPC5 KO and WT mice. Moreover, at a $0.7 \mathrm{mg}$ MIA dose administered, TRPC5 KO, but not WT mice developed weight bearing asymmetries over the course of the study and most significantly from 14 to 28 days after MIA injection. In paraformaldehyde-fixed joints obtained 28 days post MIA injection from TRPC5 KO and WT mice no significant differences in cartilage degradation or inflammatory content were observed.

Conclusions: This study suggests that deletion of the TRPC5 receptor signalling is associated with a faster onset of referred allodynia and increased guarding/ongoing pain like behaviour in a model of $O A$

\section{REFERENCES:}

[1] Xu SZ, Sukumar P, Zeng F, Li J, Jairaman A, English A, et al. TRPC channel activation by extracellular thioredoxin. Nature 2008;451(7174):6972.

[2] Alawi KM, Russell FA, Aubdool AA, Srivastava S, Riffo-Vasquez Y, Baldis sera L, Jr, et al. Transient receptor potential canonical 5 (TRPC5) protects against pain and vascular inflammation in arthritis and joint inflammation. Ann Rheum Dis 2017;76(1):252-60.

Acknowledgements: This project was supported by ARUK.

Disclosure of Interest: None declared

DOI: 10.1136/annrheumdis-2018-eular.5506

\section{SAT0052 THE COLL2-1 PEPTIDE OF COLLAGEN TYPE II: A NEW ACTOR OF SYNOVITIS IN OSTEOARTHRITIS}

C. Lambert ${ }^{1}$, D. Borderie ${ }^{2}$, F. Rannou ${ }^{2}$, Y. Henrotin ${ }^{1} .{ }^{1}$ Bone and cartilage research unit, University of Liège, Liège, Belgium; ${ }^{2}$ Inserm umr 1124, Laboratory of pharmacology, toxicology and cell signaling, University Paris Descartes, Paris, France

Background: Osteoarthritis $(\mathrm{OA})$ is characterised by degradation of the extracellular matrix associated with inadequate repair responses including pro-inflammatory pathways of nonspecific natural immune response.

Objectives: We evaluated the inflammatory effect of Coll2-1 peptide in osteoarthritic synoviocytes and rats by comparing peptide-induced inflammatory reaction with the one induced by bovine type II collagen or streptococcal cell wall.

Methods: Human synoviocytes from knee OA patients $(n=10)$ were pre-treated with AS0619 or CLI-095 (500 nM, 1 and $2.5 \mu \mathrm{M})$ before a 24 hours treatment with Coll2-1 peptide $\left({ }^{108} \mathrm{HRGYPGLDG}^{116} ; 0.45\right.$ or $\left.4.5 \mathrm{nmol}\right)$. Expression of Interleukin (IL)-8, Vascular Endothelium Growth Factor (VEGF) and phosphorylation of the IkB- $\alpha$ and p65 were evaluated. Either Coll2-1 peptide, bovine type II collagen (CIA), streptococcal cell wall (SCW) or saline solution (100 $\mu \mathrm{ISC}$ or $50 \mu \mathrm{IIA}$ ) were injected into Lewis rats $(n=108)$. The Coll2-1 peptide was subcutaneously injected (SC; 20 and $200 \mu \mathrm{g} / 100 \mu \mathrm{l} / \mathrm{animal})$ or intra-articular (IA; 0.5 and $5 \mu \mathrm{g}$ $50 \mu \mathrm{l} / \mathrm{animal})$. The bovine type II collagen was SC injected $(200 \mu \mathrm{g} / 100 \mu \mathrm{l} /$ 
animal), streptococcal cell wall in IA $(5 \mu \mathrm{g} / 50 \mu \mathrm{l} / \mathrm{animal})$. The animals were injected on day 10 and monitored for 21 or 28 days. Visual evaluation of the severity of arthritis and histological lesions were performed.

Results: Coll2-1 at $0.45 \mathrm{nmol}\left({ }^{* *} \mathrm{p}<0.01\right)$ and $4.5 \mathrm{nmol}\left({ }^{*} \mathrm{p}<0.05\right)$ significantly increased IL-8 gene expression and tended to increase VEGF expression by synoviocytes. With AS0619, a specific antiserum for Coll2-1 peptide, IL-8 expression significantly decreased $\left({ }^{*} p<0.05\right)$. Coll2 -1 also induced both translocation of $p 65$ and IkB- $\alpha$ degradation. The latter being reduced with oxidative stress inhibitors. With CLI-095, we observed a decrease of IL-8 expression. In vivo, bovine type II collagen injection and Coll2-1 peptide injection resulted in an increase in visual arthritis score from D7. The global histological score was also increased by bovine type II collagen on D21 $(p=0.0005)$ and on D28 $(p<0.0001)$ and by the peptide Coll2-1 on D21 at the concentration of $200 \mu \mathrm{g}(\mathrm{p}=0.0252)$ and on D28 at the concentration of $20 \mu \mathrm{g}(\mathrm{p}=0.0025)$. Compared to control, all the components of the histological score were similarly modified by bovine type II collagen and the peptide Coll2-1 at both on D21 and on D28: increase in the inflammatory parameter (D21 $200 \mu \mathrm{g} \mathrm{p}=0.0217$ and D28 $20 \mu \mathrm{g} \mathrm{p}=0.0021$ ), reduction of proteoglycan contents (D28 $200 \mu \mathrm{g} \mathrm{p}=0.0072$ and $20 \mu \mathrm{g} \mathrm{p}=0.0024$ ), increased cartilage degradation (J28 $200 \mu \mathrm{g} \mathrm{p}=0.0070$ and $20 \mu \mathrm{g} \mathrm{p}=0.0024$ ) and modification of the subchondral bone (D21 $200 \mu \mathrm{g} \mathrm{p}=0.0025$ and D28 $20 \mu \mathrm{g} p=0.0065$ ). Similarly, both the injection of SCW and that of Coll2-1 peptide induced an increase in the visual score from D10. The effect of Coll2-1 peptide on this score was identical to that of the SCW. Compared to control, SCW and Coll2-1 peptide increased the global histological score (D21 p=0.0119 and D28 p=0.0045). Like SCW, the injection of Coll2-1 peptide caused both inflammatory reaction, loss of proteoglycan, appearance of cartilage structural lesions (D28 $0.5 \mu \mathrm{g} \mathrm{p}=0.0201$ ) and subchondral bone modification.

Conclusions: Coll2-1 peptide is able to induce an inflammatory reaction and structural changes in articular cartilage and subchondral bone comparable to those induced by SCW and bovine type II collagen. In conclusion, Coll2-1 may initiate nonspecific natural immunity and therefore be a therapeutic target for biotherapy.

Disclosure of Interest: None declared

DOI: 10.1136/annrheumdis-2018-eular.1036

\section{SAT0053 IDENTIFICATION OF NOVEL DRUGS WITH SENOLYTIC ACTIVITY AS OSTEOARTHRITIS THERAPEUTICS}

U. Nogueira-Recalde ${ }^{1}$, F.J. Blanco ${ }^{1}$, M.I. Loza ${ }^{2}$, D. Grassi ${ }^{3}$, P. Robbins ${ }^{3}$, E. Dominguez ${ }^{2}$, B. Carames ${ }^{1} .{ }^{1}$ Rheumatology Division. Cartilage Biology Group, The Institute of Biomedical Research of A Coruña (INIBIC), A coruña; ${ }^{2}$ Biofarma Research Group, Center for Research in Molecular Medicine and Chronic Diseases (CIMUS), University of Santiago de Compostela, Santiago, Spain; ${ }^{3}$ Department of Metabolism and Aging, The Scripps Research Institute, Jupiter, FL, USA

Background: Disease-modifying treatments for Osteoarthritis (OA) are not available. Aging-related features such as failure of cellular homeostasis mechanisms, including autophagy, cause extracellular matrix damage, chondrocyte senescence and death, which leads to articular cartilage degeneration as well as loss of joint function.

Objectives: The objective of this study was to identify senolytics and activators of autophagy by cell-based imaging of approved drugs in human chondrocytes.

Methods: To induce cellular senescence and reduced autophagy, High Content Screening system. Validation assays with readouts for senescence, autophagic flux, inflammation and apoptosis in primary human chondrocytes were performed. The anabolic effect on human cartilage and chondrocytes was evaluated by Safranin O staining and Nitric oxide production. To define the effects on senescence (senomorphic or senolytic), TC28a2 chondrocytes and human lung fibroblasts (IMR90) were employed. Senescence was induced in TC28a2 and IMR90 by treatment with IL-6 $(20 \mathrm{ng} / \mathrm{ml})$ for 72 hours and Etoposide $(20 \mu \mathrm{M})$ for 48 hours, respectively, and treated with serial dilutions of identified compounds. The number of senescence cells and the number of total cells were determined with Cell Analyzer 6000 Confocal Imaging System. Navitoclax $(2,5 \mu \mathrm{M})$ and Rapamycin $(10 \mu \mathrm{M})$ were employed as reference controls for senolytic and senomorphic effects, respectively.

Results: Our primary screen yielded 279 senotherapeutic compounds. The effects of hits at inducing the autophagic flux were evaluated. 37 compounds with both senotherapeutic and pro-autophagy effects were selected. An approved drug with a defined molecular mechanism of action was selected for further validation. The compound reduced senescence $(p<0.001)$ and increased autophagic flux $(p<0.0001)$. Furthermore, we found that it protects against defective autophagy and inflammation in response to IL-6 and IL-1b. This protective effect was confirmed in human cartilage explants by a reduction of proteoglycans loss $(\mathrm{p}<0.05)$ and in primary human chondrocytes by a reduction of NO production and chondrocyte death by apoptosis $(p<0.0001)$. Moreover, a significant senolytic effect of the selected compound was observed in both chondrocytes and fibroblasts $(p<0.05)$. This effect was also observed for structurally different compounds sharing the same mechanism of action, suggesting that pharmacological modulation of this mechanism may provide therapeutic benefits in OA.

Conclusions: Our imaging screening methodology provides a unique opportunity to identify drugs and mechanisms to prevent cartilage pathology. Autophagy activation and disruption of senescence may provide benefits for delaying cartilage degeneration.

Acknowledgements:

Disclosure of Interest: None declared

DOI: 10.1136/annrheumdis-2018-eular.3680

\section{SAT0054 SIGNIFICANT DECREASE OF T-CELLS BUT NOT MACROPHAGES IN THE SYNOVIUM OF PATIENTS WITH ACTIVE RHEUMATOID ARTHRITIS AFTER TREATMENT WITH TOCILIZUMAB}

K. Chatzidionysiou, M. Engström, E. af Klint, A. Hensvold, A.I. Catrina. Department of Rheumatology, Karolinska University Hospital, Karolinska Institute, Stockholm, Sweden

Background: Tocilizumab (TCZ) is an anti-IL6R monoclonal antibody approved for the treatment of Rheumatoid Arthritis (RA). There is limited data on synovial tis sue histology changes.

Objectives: The aim of this study was to evaluate the effect of TCZ on synovial cell populations and on citrullination.

Methods: 15 patients with RA, according to ${ }^{A C R} 1987$ criteria, independent of disease duration, were included. Synovial biopsies were obtained before and after 8 weeks of treatment. We evaluated by immunohistochemistry $(\mathrm{IHC})$ expression of citrullinated proteins (CP) and protein arginine deiminase (PAD) enzymes in synovial tissue before and after treatment (1325:C03, 1325:B09, PAD2, PAD4). Negative controls were used for each antibody. Expression of CD68, CD3, CD20 and CD55 was also evaluated. Evaluation of all IHC variables was performed by two blinded independent observers using a semiquantitative score on a $0-3$ scale $(0$, no staining; 1 , low amounts of staining; 2 , moderate amounts of staining; 3 , high amounts of staining). Paired-wised Wilcoxon Signed Ranks Test was used to compare the median values.

Results: The median (IQR) age, disease duration, N. prior biologic DMARDs and DAS28 at baseline was $66,{ }^{58-79} 4(1-13), 1(0-2), 6(5-7)$, respectively. $93 \%$ were female, $53 \%$ were RF +and $60 \%$ ACPA+, $53 \%$ had concomitant glucocorticoids and only $27 \%$ had concomitant conventional synthetic DMARDs. Significant reductions in DAS28, swollen and tender joint count (SJC and TJC, respectively), and acute phase reactants (ESR and CRP) were observed between baseline and 8 weeks (table 1). By IHC, TCZ induced significant decrease in the number of CD3, C03 and CD55, but not in the number of the other CP, PAD2, PAD4 and CD68 (table 1).

Abstract Sat0054 - Table 1

Baseline 8 weeks p-value

(Wilcoxon Signed Ranks Test)

DAS28 BL 5.9 (4.7-6.8) 2.98 (2.0-3.8) 0.028

SJC BL 9 (3-14) $1(0-4.25) 0.009$

TJC BL $10^{4-15} 1(0-2.25) 0.017$

ESR BL 34 $34^{15-69} 6(5-16) 0.001$

CRP BL $11^{5-27} 1(1-2) 0.005$

CD68 2 (1-3) $2(0.75-3) 0.41$

CD3 2 (1-3) 1 (0-2) 0.046

CD55 2 (2-3) $1.5(1-2) 0.05$

C03 (cit) 1 (0-1) $0(0-1) 0.025$

B09 (cit) $2(1-2) 2(1-2) 0.20$

PAD2 $3(2-3) 2(1.5-3) 0.16$

PAD4 $2(2-3) 2(2-3) 0.37$

Conclusions: Treatment with tocilizumab reduced the number of synovial tissue T cells. This was observed in the responders but not in non-responders. Significant reduction in citrullination as assessed by expression of 1325:C03 but not 1325:B09. No significant reduction in macrophages was observed.

Acknowledgements: This study was funded by Roche.

Disclosure of Interest: None declared

DOI: 10.1136/annrheumdis-2018-eular.5459 